\title{
IoT analytics: Reaping value from loT data
}

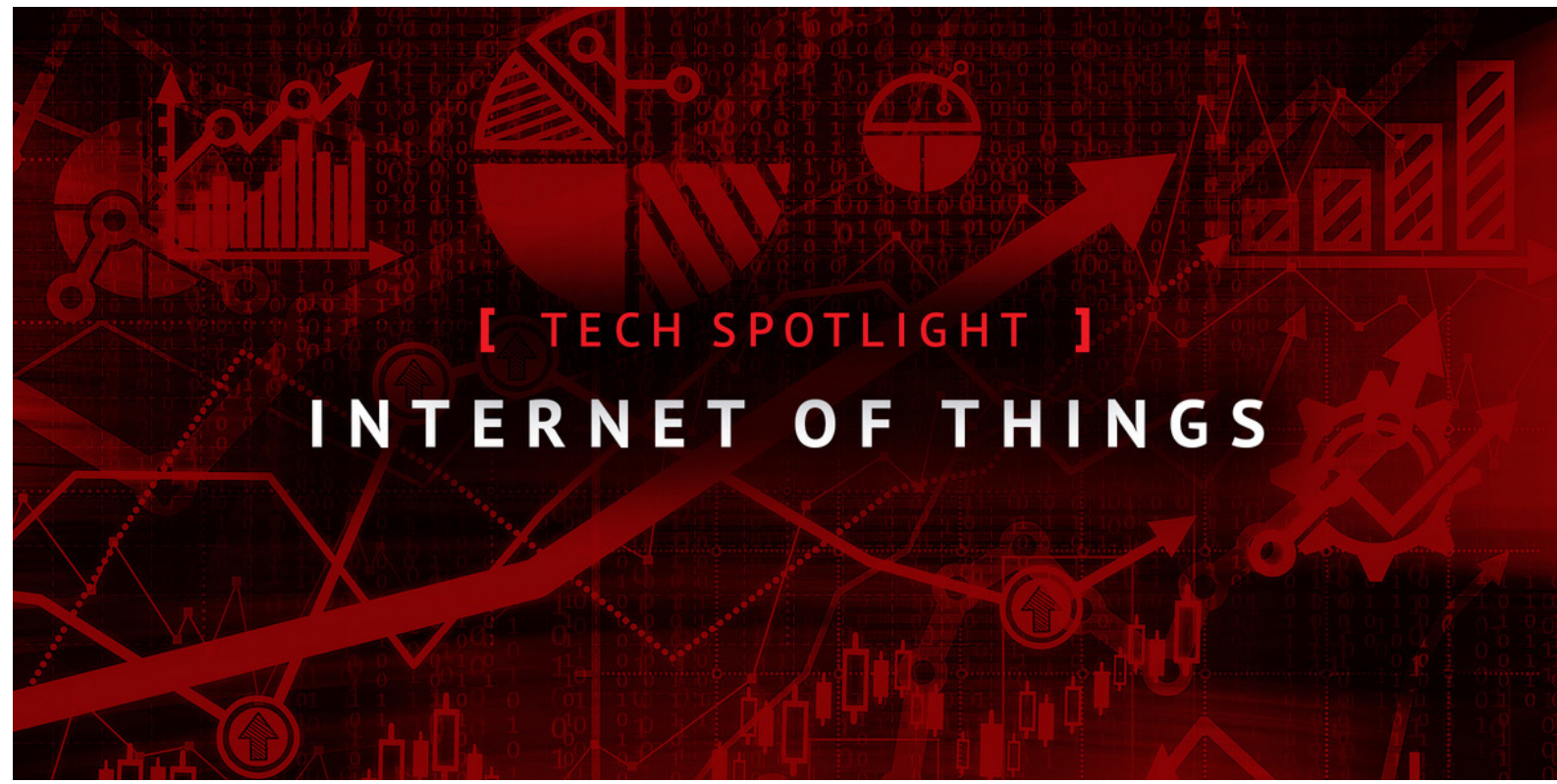

The internet of things may be your key to data-driven

\section{transformation. Here's how to turn vast troves of real-time IoT data into big-time business value.}

The internet of things (loT) is increasingly becoming a key component of many companies' data-driven transformation strategies. Indeed, organizations that have embraced IoT are already seeing benefits such as improved operational processes, better inventory management, and enhanced equipment maintenance - to name a few.

But a successful loT strategy is more than just connecting a bunch of devices and sensors to the internet and gathering data from these "things." IT must establish the ability to effectively analyze the vast amounts of data loT creates in order to make sense of it and gain real business insights.

That's why an analytics strategy for IoT should be a top priority for any company looking to get the most out of all the connectivity.

Organizations can enjoy a number of advantages in leveraging the IoT data they gather, says Carlton Sapp, senior director and research and advisory leader at Gartner.

The internet of things
How IoT is becoming the pulse of healthcare (Computerworld)

- How loT changes your threat model: 4 key considerations (CSO)

- IOT analytics: Reaping value from loT data (CIO)

- IoT down on the farm: Drones and sensors for better yields (Network World)

- How to choose a cloud loT platform (InfoWorld) These include contextual awareness of equipment and systems; improved decision-making, optimization and supervisory control of equipment and resources; reduced costs associated with data management; proactive, predictive and prescriptive management of equipment; and environmental compliance.

These opportunities are pervasive in use cases such as fleet optimization and management, asset management, financial risk management, and smart cities, Sapp says.

But they require a sound, streamlined approach to the data 
end of loT. Here are several tips for dealing with loT data, and getting the most out of these resources.

Build an IoT analytics organization and infrastructure Once an organization has an idea of its loT analytics business goals, it needs to identify the key stakeholders who will be involved, says Stacy Crook, research director for loT at IDC, and ascertain whether or those stakeholders require additional skills to make the project successful.

"It is a well-known fact that data science skills are in short supply in the industry, but these are essential for loT analytics projects," Crook says. "So the project may require hiring new employees, or outsourcing certain parts of the project to third parties," if in-house data science skills are thin.

Organizations should also consider appointing a chief data officer (CDO) to champion IoT data analytics efforts and lead the data governance strategy, Crook says.

Because loT is essentially a big data problem, IDC suggests organizations consider how their existing infrastructure could also serve loT use cases. "Although older big data architectures might have been focused on batch-oriented workloads, increasingly there are tools available to run real-time workloads over this same backbone," Crook says. Leveraging the same infrastructure for various IOT workloads can have benefits in terms of preventing data siloes and providing the ability to more easily run cross-functional data analysis across those workloads, Crook says. "It can also provide data governance and security benefits," she says.

Deploy an architecture that supports loT data growth Companies need to start with the right loT data architecture and understand how to manage loT data at various locations.

"Data emanating from loT endpoints offers new and unique challenges, such as unreliable network access and combining devices that may be distributed over large distances and generate data in multiple formats over multiple protocols," Sapp says.

Today, most loT data is telemetry data, but endpoints are increasingly emitting image and audio data that should be handled by persistent data stores, Sapp says. "Start with an appropriate IOT data architecture that will support the expected growth in the volume of IoT," he says.

Organizations often fail to effectively manage loT data, due to a lack of a flexible/elastic data architecture. "Data will continue to grow, so design an architecture that leverages analytics and data mining techniques that identify critical information that can be used to improve processes, improve decision-making, or reduce costs," Sapp says.
For example, telecommunications companies are successful at reducing the cost of moving data over a network by taking advantage of loT analytics at the network edge that reduces "noisy data."

"Those organizations focus on scalable edge-centric data architectures that are designed for rapid knowledge discovery in loT data," Sapp says.

Deliver analytics across data pipelines

The IOT data architecture should also support analytics across data pipelines (via streaming) and in local data stores to take advantage of faster decision-making and reduced costs, Sapp says.

Organizations can do this by focusing on data-centric design patterns when creating and deploying loT analytics, including the use of event-driven architectures.

"Start by distributing analytics at the edge, on streaming pipelines, on the platform, and in the enterprise," Sapp says. Organizations should take advantage of streaming loT data pipelines as a source to deploy analytics to improve latency and reduce costs and security vulnerabilities, he says.

For example, the U.S. Department of Defense often performs analytics over streaming data pipelines to reduce the throughput of data over a network, Sapp says. It also leverages loT edge analytics to avoid sending any data over a network, using operational analytics closer to the source of data.

There will most likely be multiple analytical environments deployed to support disparate analytics, Sapp says. "Environments may range from operating systems to embedded analytics software," he says. "Be prepared to deploy loT analytics across a landscape that spreads from the network edge to the corporate enterprise. For example, utility organizations leverage distributing loT analytics across various infrastructures to support fleet management."

Leverage artificial intelligence

Organizations should enhance what they can do with loT data by taking advantage of $\mathrm{Al}$, Sapp says.

"Edge intelligence is an emerging field that uses $\mathrm{Al}$ as an analytic method deployed at the network edge, to develop intelligent applications from loT data," Sapp says.

These intelligent applications range from video surveillance to intelligent supervisory control and data acquisition (SCADA) systems. For example, environmental organizations use IoT data to build intelligence control systems to maintain environmental compliance.

Adding $\mathrm{Al}$ to the IoT architecture Is becoming an operational imperative, Sapp says. loT systems, including endpoint devices, must become smarter and more autonomous in order 
to deal with the ever-increasing magnitude of data. To make these systems smarter, organizations need to deploy $\mathrm{Al}$ and machine learning.

Be a cloud native

Given the huge volumes of data generated by IoT applications, for many organizations the cloud will be the only answer for getting a hold on data management, including analytics.

"It's not worth it to build the scale and speed needed to really manage this volume in real time," says Greg Meyers, group $\mathrm{CIO}$ and chief digital officer at Syngenta, a company that produces agrochemicals and seeds.

"Trying to manage it yourself in your own data center or on your own infrastructure is hugely self-defeating," Meyers says.

IOT gives Syngenta the ability to manage its customers' farms and fields, which are usually arbitrarily aggregated into small micro segments. "Humans are great at managing averages, but computers are better at managing variability," Meyers says. "IOT lets us understand why things that are happening in one area are different than things that are happening maybe 100 meters away."

Leading public cloud vendors are offering services to help companies with IoT analytics. For example, Amazon Web Services (AWS) offers loT Analytics, a managed service that enables companies to run and operationalize sophisticated analytics on massive volumes of IoT data, without having to worry about the cost and complexity typically required to build an loT analytics platform.

Microsoft offers Azure IoT, which includes a data analytics service called Azure IoT Central to provide analytics capabilities to examine historical trends and correlate various telemetries from connected devices. And Google provides Cloud IoT, a set of tools to connect, process, store, and analyze data both at the network edge and in the cloud.

Prioritize data governance, security, and privacy

Organizations need to ensure they have governance, security, and privacy mechanisms in place for loT data analytics processes. Much of the data generated by loT will be sensitive or have competitive value - and needs to be carefully managed and protected.

"Reassess current data governance practices [to] include machine data," says Nicholas Colisto, vice president and $\mathrm{CIO}$ at Avery Dennison, a manufacturer and distributor of adhesive materials, apparel branding labels, and tags.

"From my experience, loT governance is an immature area," Colisto says. "In a previous company, I faced a situation where a business unit deployed an loT system without seek- ing IT involvement, and simple operational tasks and tools to audit devices and apply firmware were not considered."

Companies need to consider loT data risks based on confidentiality, privacy, and retention requirements, Colisto says. "For example, if you are working with personal data, consider the issues that can arise from algorithmic bias or inability to comply with regulations such as GDPR [General Data Protection Regulation], which can lead to legal action and damage your company's reputation," he says.

Leverage loT data for new revenue opportunities

Data generated from loT can be valuable both inside and outside the company.

Chemical manufacturing company Texmark Chemicals launched an effort to modernize operations at its plant by deploying sensor-enabled pumps. Using technology from Hewlett Packard Enterprise and Aruba Networks, the company gathers operational data from pump sensors that measure temperature, pressure, vibrations, flow, and power. This data is analyzed to predict equipment failures before they happen.

Through a "workshopping" process, Texmark realized that having sensor-enabled equipment not only helps the company monitor its assets and processes, but has opened the possibility to new business models, says Doug Smith, CEO. The use of IoT becomes an additional selling factor prior to contract negotiations, Smith says. "Clients are beginning to realize the value of having access to data coming off contractor assets," such as industrial pumps, he says. Clients then ask Texmark to add sensors to their pumps and provide them with the data.

"In essence, we are developing a library of historic performance attributes that can be catalogued and shared with other companies using similar equipment," Smith says. "When deploying machine learning analytical models, the more data acquired, the greater the accuracy in the analytical prediction."

By sharing loT data with pump manufacturers or fellow suppliers, "we could prove the new business model, as long as the documentation is clear and precise," Smith says. "Meanwhile, customers are impressed we have deployed instrumentation and software analytics to capture, analyze, and report on such data - allowing for more cost-effective decisions."

This new data-as-a-service offering enabled by loT can distinguish Texmark from competitors, Smith says, and creates a stronger bond with customers while empowering employees to achieve more from their work. 